\title{
DEVELOPMENT OF A NON-DESTRUCTIVE BEAM PROFILE MONITOR USING A GAS SHEET
}

\author{
Y. Hashimoto, Y. Fujita, T. Morimoto, S. Muto, KEK, Tsukuba, Japan \\ T. Fujisawa, T. Honma, K. Noda, Y. Sato, S. Yamada, NIRS, Chiba, Japan \\ H. Kawauchi, A. Morinaga, Y. Taki, Science University of Tokyo, Chiba, Japan \\ K. Takano, J. Takano, Takano Giken CO, Ltd., Kanagawa, Japan
}

\section{Abstract}

A non-destructive beam profile monitor using a neutral gas sheet is developed for ion synchrotron beams.

To produce the intense gas sheet, a nozzle beam method is employed. The typical sizes of the sheet are 100 $\mathrm{mm}$ width and $1 \mathrm{~mm}$ thickness. Up to now the pressure of the gas sheet has been achieved $5 \times 10^{-7}$ Torr. As the sheet is inclined 45 degrees in the transverse direction to the beam, a cross-sectional profile of the beam would be measured.

As a performance test, a profile of an extracted proton beam from NIRS-Cyclotron was successfully measured.

\section{INTRODUCTION}

We try to develop a very fast monitor by using highdensity gas sheet ${ }^{1,2}$. Two methods are considered to detect profiles according to ion beam intensity. On the case of relatively lower intensity machine such as the NIRSHIMAC synchrotron, generated ions from the gas target are used for detecting beam profile. On the other hand, on the case of high intensity machine such as the $50 \mathrm{GeV}$-PS of JAERI-KEK joint project (JKJ), the method of observing a luminescence light from the sheet directly is put up as a candidate. In this paper ionization method is described mainly.

To generate an intense molecular beam is essential for the monitor. So the nozzle beam method is employed ${ }^{3,4}$. By using this method, it is possible to obtain several thousand times as intense as conventional molecular source. Its flux intensity becomes about $10^{19}$ molecules/sr.sec ${ }^{3,4}$.

The thickness of the gas sheet becomes thinner, the ionization point is determined more clearly. In such a point of view, a high gradient magnetic field is applied to an oxygen molecule (magnetic moment: 2.0 BohrMagneton, spin: 1) to be focused.

\section{EQUIPMENT}

\subsection{Beam Profile Measurement System}

Layout of the beam profile detector ${ }^{1}$ is shown in Fig. 1. Ions generated by the beam at the inclined sheet beam target are collected with a radial electric field induced by semi-spherical electrodes and multiplied by a two stages MCP (Hamamatsu F2226: max. gain $1 \times 10^{7}$ ). The luminescence light from a phosphor screen (decay time of the light : $100 \mathrm{~ns}$ at $1 / 10$ ) positioned just downstream of the MCP is detected by a CCD camera attached an image intensifier (I.I.: max. gain $1 \times 10^{4}$ : Hamamatsu C4078). Using radial electric fields a picture is gained geometrically about 3.0 times at the front of the MCP.

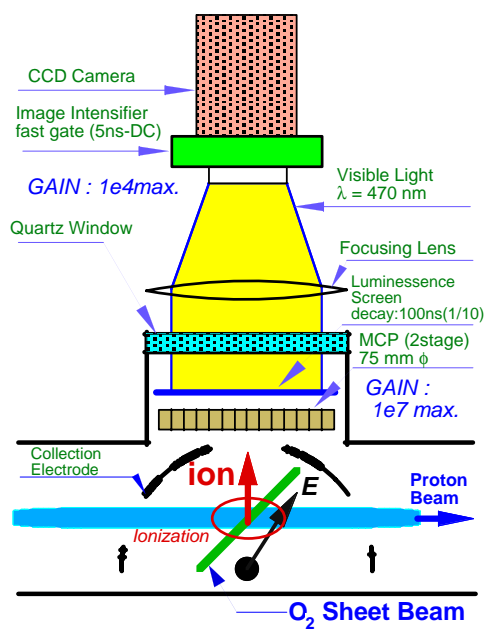

Fig. 1 Layout of the Monitor Head.

\subsection{Over View of the Sheet Beam Generator}

The sheet generator is composed of differential pumping system with five chambers (see Fig. 2). On each chamber turbo molecular pump having ability about 1000 $\mathrm{L} / \mathrm{s}$ is employed. The sheet beam generated by the nozzle and the skimmer in the gas jet chamber, runs to the detector chamber through the slit chamber and the magnet chamber. In the slit chamber, the diverged molecules are rejected and evacuated, and in the magnet chamber an oxygen sheet beam is deflected to increase the intensity at the median plane with the multi-pole magnet. In the monitor chamber the sheet beam collides with an ion beam to detect the beam profile, and in the detector chamber the profile and the intensity of the sheet beam itself are measured using compression gauge ${ }^{1}$.

Present status of the sheet beam is about $100 \mathrm{~mm}$ width and $1.3 \mathrm{~mm}$ thickness (FWHM) at the beam colliding point. These values are adapted geometrically from obtained ones at the detector chamber. The spatial uniformity is almost within $3 \%$.

\section{Nozzle-Skimmer}

As molecules are made done adiabatic free expansion in the space between the nozzle and the skimmer, the molecules have supersonic velocities and cooled. They become a molecular beam passing through the collimation slit. It has been tried to increase intensity of the molecular beam by improvement of skimmer shape 


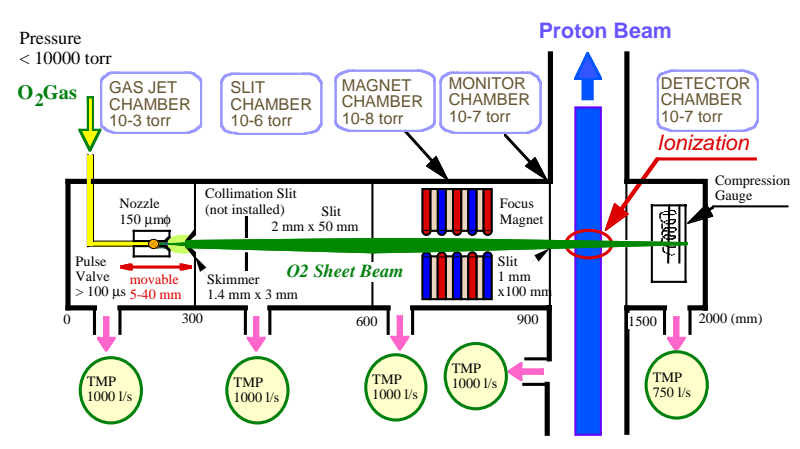

Fig. 2 Schematic view of the neutral gas sheet generator.

and size of nozzle diameter. Optimum skimmer until now has sizes of $1.4^{\mathrm{V}} \times 3^{\mathrm{H}}$ slit and outer blade angle of 60 degree and length of about $50 \mathrm{~mm}$. Optimum nozzle diameter is $150 \mu \mathrm{m}$. Employing these skimmer and nozzle, when operating source pressure is about 6000 Torr, sheet beam intensity without magnet was recorded 5 x $10^{-7}$ Torr at the beam colliding point with compression gauge method ${ }^{1}$. Obtained intensity is from 5 times to 1 order lower than the design value. Near future to increase the intensity reinforcement of pump ability of the gas jet chamber and trying to use a multi-hole nozzle are planed.

In this system molecular beams were pulsed with an electro-magnetic valve $(>100 \mu \mathrm{s})$ operation positioned before nozzle. The supplying gas flow is regulated within $1 \%$ by mass flow system (MKS 250E, 670A).

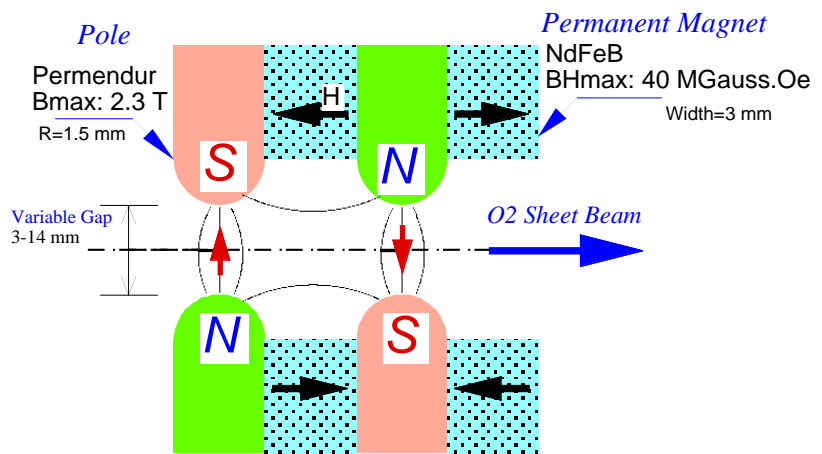

Fig. 3 Layout of one cell of the focusing magnet.

\section{Focusing Magnet}

The layout of the focusing magnet for an oxygen molecular beam is shown in fig. 3. It is compose with permanent magnets $(\mathrm{NdBFe})$ and high permeability material (Permendur) for the poles. Its number of the pairs of the poles is 26 and its total sizes are $153 \mathrm{~mm}$ long and $70 \mathrm{~mm}$ wide. Controlling the field strength the pole gap is variable in a range of 3 to $14 \mathrm{~mm}$. In case of $5 \mathrm{~mm}$ pole gap, it generates a magnetic field of about 1 Tesla at the tip of the pole. Its calculated mean gradient field in a vertical direction giving kick force to the beam is amount to about $4 \times 10^{4}$ gauss $/ \mathrm{cm}$. The effect on the oxygen beam was measured (see fig. 4). In this data comparing magnet off (gap $=14 \mathrm{~mm}$ ) to on (gap $=7 \mathrm{~mm}$ ), the beam intensity at the center becames about 2 times by focusing effect on oxygen molecule beam. So it estimated 2.3 times by calculation $^{1}$, the obtained effect seems to be consistent.

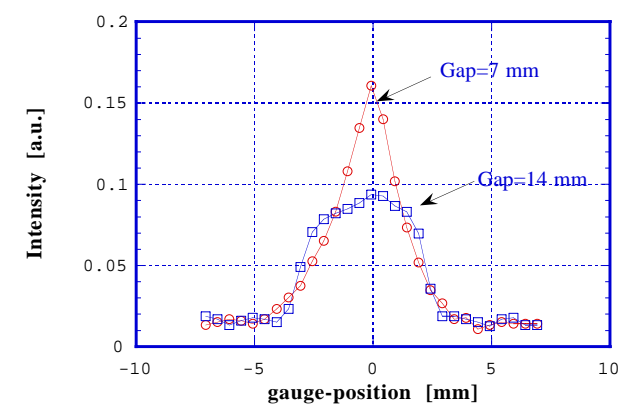

Fig.4 Focusing Effect on the Oxygen Beam.

\section{BEAM TEST}

Beam profile measurement test has been done with 8 $\mathrm{MeV}$ proton beams extracted from the NIRS cyclotron. A profile image of the beam whose mean current is $5 \mu \mathrm{A}$ (see fig. 5 (a)) was measured. In this case the gate time of the I.I was $5 \mathrm{msec}$. Obtained raw data having not only the effect of the gas sheet $\left(\mathrm{N}_{2}: 3.5 \times 10^{-7}\right.$ Torr) but also the effect of ionized residual gas $\left(5 \times 10^{-8}\right.$ Torr) along the beam path, to only see the effect of the gas sheet, the data of the gas sheet off was subtracted from the data (raw data) of it on. And its projection data of horizontal and vertical direction are shown in fig. 5 (b). About beam sizes are $5 \mathrm{~mm}^{\mathrm{H}}$ and $4 \mathrm{~mm}^{\mathrm{V}}$ respectively (FWHM) estimated by the beam sizes detected with a destructive type profile monitor positioned down stream.
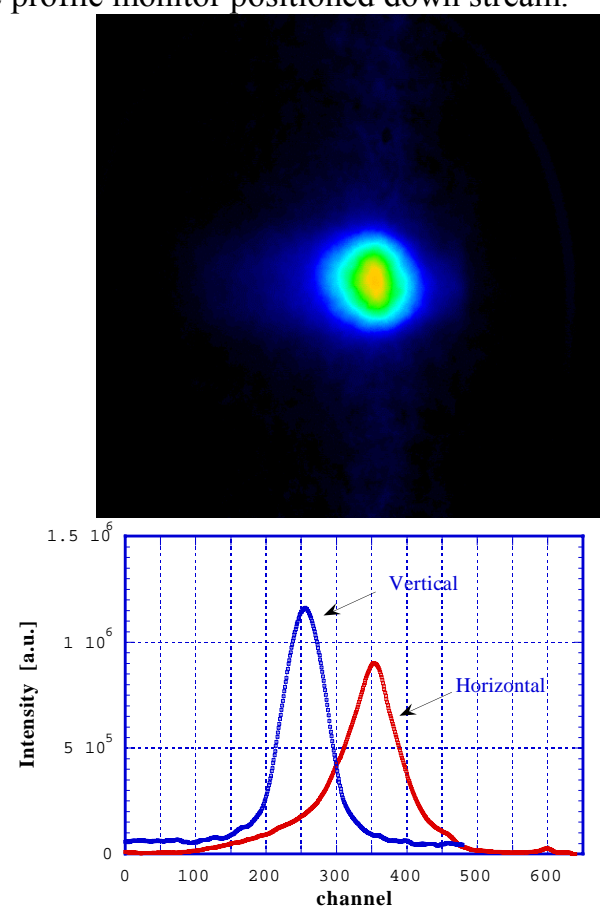

Fig. 5 (a) Measured beam profile image of an $8 \mathrm{MeV}$ proton beam of its current is $5 \mu \mathrm{A}$. (b) Its projected profile data.

Under the condition of $5 \mu \mathrm{A}$ mean current, it is examined how shortened the gate time of the I.I is. 
Obtained minimum I.I. gate time was $300 \mu \mathrm{sec}$ for a meaningful profile data (see fig. 6).
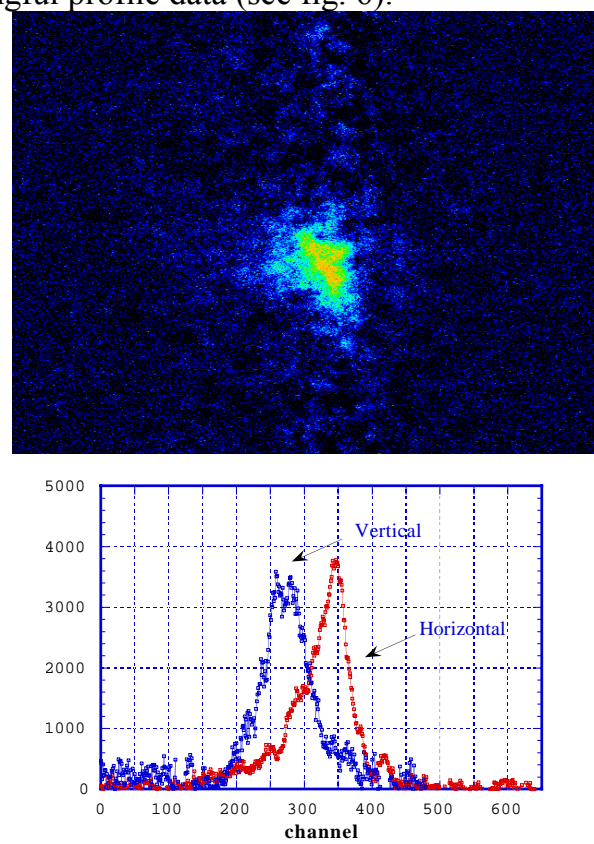

Fig. 6 (a) A profile image with $300 \mu$ sec I.I. of an $8 \mathrm{MeV}$ proton beam whose mean current is $5 \mu \mathrm{A}$. (b) Its projected profile data

It is scheduled fast beam profile measurement on the NIRS-HIMAC synchrotron using this instruments in next fiscal year. So yields for its synchrotron beam was simply estimated with the data of minimum I.I. gate time. Although the HIMAC synchrotron is relatively low intensity machine, it is hope to get higher yields so heavy ions (mainly ${ }^{12} \mathrm{C}^{6+}$ ) are accelerated from injection energy of $6 \mathrm{MeV}$ to $800 \mathrm{MeV}(270 \mathrm{MeV})$. The estimation was done with multiplying $\mathrm{N}_{\mathrm{b}}$ by $(\mathrm{dE} / \mathrm{dx})_{\mathrm{p}}$. Here $\mathrm{N}_{\mathrm{b}}$ is the number of particle passing the gas target and $(\mathrm{dE} / \mathrm{dx})_{\mathrm{p}}$ is the value of the energy loss par particle which was calculated by the Bethe-Bloch formula ${ }^{5}$. The summary is shown in Table 1. It is able to consider that single bunch measurement is possible at the time of HIMAC injection. At higher energy region of such as $270 \mathrm{MeV}$, it seems taking a meaningful profile data requires several beam bunches. Note that on this measurement margins of the MCP and I.I. gain were remains, so more experiments under their higher gains condition are needed for accurate yield estimation.

Table 1 Estimating yields for HIMAC synchrotron beam.

\begin{tabular}{ccc}
\hline & $\begin{array}{c}\text { Cyclotron } \\
(\text { Proton } 8 \mathrm{MeV})\end{array}$ & HIMAC $\left({ }^{12} \mathrm{C}^{+6}\right)$ \\
\hline $\mathrm{N}_{\mathrm{b}}$ & $1 \times 10^{10} / 300 \mu \mathrm{sec}$ & $2.5 \times 10^{8}$ particles $/$ bunch \\
$-(\mathrm{dE} / \mathrm{dx})_{\mathrm{p}}$ & $3.8 \times 10^{-11} \mathrm{MeV} / \mathrm{cm}$ & $1.74 \times 10^{-9} \mathrm{MeV} / \mathrm{cm}(6 \mathrm{MeV})$ \\
& & $9.52 \times 10^{-11} \mathrm{MeV} / \mathrm{cm}(270 \mathrm{MeV})$ \\
$\mathrm{N}_{\mathrm{b}} \times(\mathrm{dE} / \mathrm{dx})_{\mathrm{p}}$ & $3.8 \times 10^{-1} \mathrm{MeV} / \mathrm{cm}$ & $4.35 \times 10^{-1} \mathrm{MeV} / \mathrm{cm}(6 \mathrm{MeV})$ \\
& & $2.38 \times 10^{-2} \mathrm{MeV} / \mathrm{cm}(270 \mathrm{MeV})$ \\
\hline
\end{tabular}

On another experiment such as detection of luminescence light from $\mathrm{N}_{2}$ gas ${ }^{6}$ using proton $8 \mathrm{MeV}$ beam was started. The purpose is fast beam profile measurement of future JKJ-PS (3-50 GeV). In that machine as the beam intensity amounts to $2 \times 10^{13}$ protons/bunch, it is difficult to detect ionized particles accurately by disturbing induced field around such a dense charged particle beam.

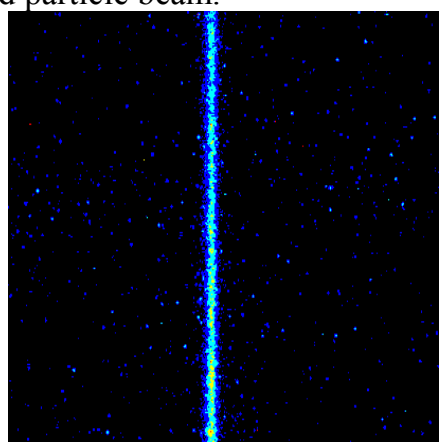

Fig. 7 A profile image of an $8 \mathrm{MeV}$ proton beam of its current is $10 \mu \mathrm{A}$ using luminescence light from $\mathrm{N}_{2}$ gas filling in the monitor chamber whose pressure is $5 \times 10^{-4}$ Torr.

Used experiment set is the thing removed MCP and screen from the set shown in fig. 5. And no voltages were operated to the electrodes. Moreover not the gas sheet but the filling $\mathrm{N}_{2}$ gas in the monitor chamber was used. The reason of using $\mathrm{N}_{2}$ gas is that higher luminescence yield and short decay time (life is 10 nsec order) are reported ${ }^{6}$.

Preliminary result is shown in fig. 7. This data was taken with the conditions that the pressure of $\mathrm{N}_{2}$ is $5.0 \times 10^{-4}$ Torr and the proton beam current is $10 \mu \mathrm{A}$. Used integration time is one frame $(1 / 30 \mathrm{sec})$. The yields from this data is seemed rather lower than the luminescence profile monitor of the CERN-SPS ${ }^{6}$. Though considered reasons are lower camera (I.I.) gain and the effect of noise integration (taking longer integration time because of using low intensity beam), they are not clearly. Hereafter more precise experiment will be done with this set and using high intensity proton beam $\left(10^{12}\right.$ protons/bunch order) at KEK PS (12 GeV) extracted beam line.

\section{FUTURE PLAN}

(1) Trying to increase the gas jet density about 5-10 times. (2) Installing the instrument to the NIRS-HIMAC synchrotron (March/2002 scheduled). And studying fast beam profile measurement with lower intensity beams by ionization detection. (3) Studying with luminescence light emitted from excited gas sheet for the JHF $50 \mathrm{GeV}$-PS.

\section{REFERENCES}

[1]Y.Hashimoto, et al., EPAC2000, Vienna, p1729-1731

[2]R. Galiana, et al. CERN, PS 91-29 (OP).

[3] J. Ross, "Molecular Beams", John and Wiley \& Sons, New York, 1966.

[4] A. Kantrowitz and J. Grey, Rev. Sci. Instr., 22, 328 (1951).

[5] W.R.Leo, Techniques for Nuclear and particle Physics Experiments, Springer-Verlag, 1987, p24.

[6] G. Burtin, et al., EPAC2000, Vienna, p256-258 